Meta

Journal des traducteurs

Translators' Journal

\title{
Cerveau et langage : nouvelles perspectives
}

\section{Yves Joanette et André Roch Lecours}

Volume 29, numéro 1, mars 1984

Cerveau, langage et traduction

URI : https://id.erudit.org/iderudit/003438ar

DOI : https://doi.org/10.7202/003438ar

Aller au sommaire du numéro

Éditeur(s)

Les Presses de l'Université de Montréal

ISSN

0026-0452 (imprimé)

1492-1421 (numérique)

Découvrir la revue

Citer cet article

Joanette, Y. \& Lecours, A. R. (1984). Cerveau et langage : nouvelles perspectives. Meta, 29(1), 27-35. https://doi.org/10.7202/003438ar d'utilisation que vous pouvez consulter en ligne.

https://apropos.erudit.org/fr/usagers/politique-dutilisation/ 


\section{CERVEAU ET LANGAGE : NOUVELLES PERSPECTIVES}

Yves JOANETTE ET ANDRÉ Roch LECOURS

Les premières conceptions modernes de l'organisation fonctionnelle du cerveau pour le langage ont vu le jour vers la fin du siècle dernier. Il a été montré dans un autre article (voir chapitre précédent) comment l'étude des troubles acquis du langage faisant suite à la survenue d'une lésion cérébrale - l'aphasie - avait permis d'entrevoir quels étaient les sous-bassements neurobiologiques des comportements de communication que représentent le langage oral et le langage écrit. C'est ainsi qu'il est apparu que l'une des caractéristiques fondamentales de cette organisation fonctionnelle est sa représentation asymétrique au sein du substrat neurobiologique, en l'occurrence les hémisphères cérébraux. Depuis les écrits de Marc Dax et de Paul Broca, on sait en effet que l'organisation fonctionnelle du cerveau pour le langage est latéralisée, le plus souvent en faveur de l'hémisphère gauche. On sait aussi qu'à l'intérieur même de l'hémisphère gauche, la contribution fonctionnelle de chacune de ses parties à l'organisation et à l'actualisation des comportements de communication est loin d'être en tous points égale. Certaines structures cérébrales - et particulièrement certaines régions du cortex - apparaissent comme plus importantes que d'autres pour sous-tendre certains aspects particuliers de la fonction linguistique; comme il a été vu précédemment, ces régions cérébrales sont globalement désignées sous l'appellation de "zone du langage" (voir chapitre précédent). Très tôt, il est aussi apparu que certains paramètres pouvaient influencer l'organisation fonctionnelle du cerveau pour le langage, ou tout au moins y être fortement corrélés. C'est ainsi qu'il est apparu que le degré d'asymétrie fonctionnelle, de même que la latéralisation de cette asymétrie, diffère selon la prévalence manuelle et selon la présence ou l'absence d'une histoire familiale de gaucherie manuelle ou d'ambidextérité. Ces paramètres sont le reflet de prédispositions innées inscrites dans le bagage génétique de chaque individu. D'autres paramètres susceptibles d'influencer l'organisation fonctionnelle du cerveau pour le langage ont été cités dans l'article précédent ; rappelons ici l'ensemble des paramètres environnementaux dont la liste non exhaustive comprend à ce jour le degré de scolarisation, la connaissance d'une ou de plusieurs langues secondes, le caractère tonal ou atonal de certaines langues, ou encore la nature de leur code écrit. Bien que l'ensemble des facteurs génétiques ou environnementaux soit désormais cité de manière relativement systématique - voire "classique " - lorsqu'on parle aujourd'hui de l'organisation fonctionnelle du cerveau pour le langage, leur rôle précis de même que leurs liens avec l'organisation cérébrale ne sont qu'à peine entrevus. De même, la liste des paramètres relevant de facteurs génétiques ou environnementaux dont on dispose aujourd'hui ne peut être considérée comme exhaustive.

En dépit du très grand nombre de questions encore laissées sans réponse et d'une évidente impossibilité d'intégrer dans un seul modèle de l'organisation fonctionnelle du cerveau pour le langage l'ensemble des données aujourd'hui disponibles, il s'ouvre de nouvelles perspectives de recherches qui ajouteront probablement encore d'autres facet- 
tes à inclure dans toute quête des liens mutuels entre cerveau et langage. Notre but est ici d'évoquer certaines de ces nouvelles perspectives de recherche en tentant de montrer en quoi les portes qu'elles ouvrent devraient permettre d'entrevoir avec plus de justesse l'organisation fonctionnelle du cerveau pour le langage.

\section{LA DIMENSION CHRONOBIOLOGIQUE}

Jusqu'à tout récemment, la dimension chronobiologique - la prise en compte du facteur "temps" - n'avait que peu de place dans les modèles de l'organisation fonctionnelle du cerveau pour le langage. En fait, cette dimension n'était prise en compte que lors de cette première époque de la vie qui prend fin avec l'accession à l'âge adulte et pendant laquelle s'effectue l'ontogénèse, c'est-à-dire le développement de l'ensemble des fonctions telles qu'elles apparaissent chez l'adulte. C'est l'étude des aphasies acquises de l'enfant lors de son développement qui a permis de décrire la dynamique ontogénétique de cette organisation fonctionnelle, tant dans sa répartition interhémisphérique que dans son organisation intrahémisphérique. En effet, les enseignements désormais classiques (Alajouanine \& Lhermitte 1965; Basser 1962; Hécaen 1976) complétés par des données plus récentes (Vargha-Khadem et al. 1963) nous apprennent premièrement que l'aphasie acquise de l'enfant peut survenir plus fréquemment que chez l'adulte à la suite d'une lésion hémisphérique droite, du moins jusqu'à un certain âge. Cet âge s'est progressivement abaissé avec les années et il est aujourd'hui admis qu'une telle situation ne prévaut probablement que lors de la petite enfance. Dès l'âge de 2-3 ans, les lésions aphasiogènes sont de plus en plus latéralisées à gauche. Ainsi, de non latéralisée qu'elle est lors de la petite enfance, l'organisation fonctionnelle du cerveau pour le langage se latéralise peu à peu au cours de l'enfance et de l'adolescence. Ce processus de latéralisation se fait toutefois lentement et progressivement comme en témoigne la récupération surprenante d'une aphasie acquise par lésion cérébrale gauche lors de l'enfance, et même lors de l'adolescence. Ce dernier fait nous permet de supposer que, malgré ce processus de latéralisation, l'hémisphère cérébral controlatéral continue à pouvoir soustendre certains aspects de la fonction linguistique et, partant, est à même de pouvoir prendre la relève si une lésion survient.

Le deuxième principe que ces données nous enseignent est la surprenante régularité de la séméiologie de l'aphasie acquise chez l'enfant. En effet, le tableau clinique habituellement réalisé est celui d'une aphasie de $\mathrm{Broca}^{1}$ et ce, quel que soit le site lésionnel. Il semble donc que la fonction linguistique ne s'organise de manière spécifique au sein de la "zone du langage" que progressivement au cours de l'enfance.

Les données dont on dispose aujourd'hui nous permettent de croire que la dynamique ontogénétique identifiée chez l'enfant ne fait pas place à une stabilité absolue de l'organisation fonctionnelle du cerveau pour le langage lors de la vie adulte comme pouvait le laisser croire un enseignement traditionnel. En effet, il est désormais admis que, chez l'adulte, une même lésion cérébrale puisse être la cause d'une séméiologie aphasique différente selon l'âge (Brown 1982; Joanette et al. 1983; Obler et al. 1978). C'est ainsi que la proportion des aphasies de "type Broca ", de maximale qu'elle était lors de l'enfance, tend à diminuer tout au long de la vie adulte, alors que celle des aphasies de "type Wernicke "tend plutôt à augmenter. D'après Poncet et Ponzio (1975), la forme la plus prototypique de ce dernier type d'aphasie - l'aphasie temporale complète d'Alajouanine (1968) - surviendrait essentiellement au cours de la dernière période de la vie,

1. Le lecteur pourra trouver dans le chapitre précédent une définition des aphasies de Broca et de Wernicke. 
après 70 ans. Les observations d'aphasie de Wernicke avec glossolalie rapportées par Lecours et al. (1981) et Perecman et Brown (1981) vont dans le même sens.

Ces faits nous incitent à penser que l'organisation fonctionnelle du cerveau pour le langage n'est pas un processus qui se stabilise au cours de la vie adulte mais bien un processus qui a une dimension chronobiologique omniprésente. Mais comment rendre compte d'une telle dimension? Seuls quelques rares modèles de l'organisation cérébrale ont tenté de l'intégrer. Le modèle de Jason Brown représente l'une des tentatives du genre (Brown 1976; Brown et Hécaen 1976; Brown 1982). Postulant que l'évolution observée chez l'adulte est dans la lignée de l'évolution notée chez l'enfant au cours de l'ontogénèse, il suppose que l'organisation du cerveau pour le langage subit une " concentration fonctionnelle " au sein de la "zone du langage" de l'hémisphère gauche. Autrement dit, il y aurait une restriction progressive du substrat neurobiologique soustendant la fonction linguistique. Une telle restriction concernerait tant la répartition interhémisphérique (processus de concentration progressive au sein de la "zone du langage $»$ ). Selon Jason Brown, cette restriction fonctionnelle impliquerait certains aspects de la fonction linguistique avant d'autres. Par exemple, les processus liés à l'expression (encodage) se latéraliseraient et se concentreraient plus précocement que ceux liés à la compréhension (décodage) ; de même, les processus situés plus en aval dans un modèle du fonctionnement linguistique (par exemple, la réalisation phonologique) se latéraliseraient et se concentreraient plus précocement que des processus situés plus en amont (par exemple, les processus lexico-sémantiques). C'est cette dynamique constante, présente tout au long de la vie, qui serait pour Jason Brown à l'origine du fait précédemment mentionné qu'une même lésion cérébrale acquise peut être à l'origine de comportements aphasiques différents selon l'âge. Ainsi, si de tels modèles se vérifient, les relations mutuelles entre cerveau et langage devront dorénavant prendre en considération cette quatrième dimension, et ce, même au cours de la vie adulte. Il devient donc souhaitable d'inclure une telle dimension chronobiologique dans tout modèle qui tente de décrire l'organisation fonctionnelle du cerveau pour le langage.

\section{"SYSTÉMATIBILITÉ " ET "ROUTINISATION"}

Cette seconde avenue de recherche n'est pas sans lien avec la première évoquée, c'est-à-dire la dimension chronobiologique. En effet, nous avons vu comment l'organisation fonctionnelle du cerveau pour le langage semble en constante transformation, entraînée qu'elle est par une dynamique qui la pousse à se spécifier de plus en plus au sein de son substrat neurobiologique. Le modèle explicatif de Jason Brown auquel nous avons précédemment fait allusion ne fournit pas les raisons qui pourraient expliquer une telle dynamique. En fait, Jason Brown semble postuler que cette dynamique est plus ou moins préprogrammée, c'est-à-dire chronobiologique au sens génétique du terme. Un tel postulat rend probablement compte d'une partie non négligeable de ce phénomène. Toutefois, si le " temps qui passe » peut être le reflet d'une dynamique inscrite dans le bagage génétique, il convient de remarquer qu'il correspond aussi à une augmentation - soit uniquement quantitative, soit à la fois quantitative et qualitative - de l'exposition au milieu environnant. C'est ainsi qu'il existe aujourd'hui d'autres modèles - en un sens plus dynamique - du fonctionnement cérébral qui ont pour point de départ les caractéristiques d'une tâche donnée et le degré d'utilisation de cette tâche. Le modèle proposé par Goldberg et Costa (1981) est un modèle du fonctionnement cérébral, linguistique ou autre, qui va dans ce sens. Il repose sur une conception du fonctionnement cérébral où l'hémisphère droit est présenté comme celui qui met sur pied, qui élabore le "système descriptif » — ou " code" — nécessaire pour réaliser une tâche donnée alors que l'hémisphère gauche est plutôt celui qui est apte à appliquer un système descriptif 
déjà connu. Ainsi, les réseaux neuroniques mis en branle pour la réalisation d'une tâche donnée dépendent (a) de la possibilité d'établir un système descriptif qui permette de réaliser cette tâche (sa "systématibilité ") et (b) du degré d'utilisation d'un système descriptif donné (sa "routinisation"). Plus une tâche donnée est systématisable et qui plus est routinisée, plus elle sera sous-tendue par des réseaux neuroniques spécifiques de l'hémisphère gauche. Le premier avantage d'un tel modèle est qu'il est valable pour toute tâche et pour toute fonction, que ce soit la compréhension du langage, l'expression musicale, la reconnaissance des visages ou autres. Le second avantage est qu'il ne fixe pas de façon canonique l'organisation fonctionnelle du cerveau pour une fonction donnée ; cette organisation sera tributaire du degré de systématibilité et de routinisation d'une $t \hat{a}$ che particulière chez un individu donné à un moment précis de son cheminement personnel.

La fonction linguistique représente une fonction parmi les plus systématisables puisqu'elle constitue en soi un système descriptif - un code - conventionnellement accepté par une communauté donnée. C'est aussi l'une des premières fonctions qui a été associée au fonctionnement de l'hémisphère gauche et l'une de celles qui dépend le plus de son intégrité. De plus, la latéralisation progressive vers l'hémisphère gauche au cours de l'ontogénèse et au cours de la vie adulte pourrait refléter le fait qu'une fois élaboré, le système descriptif linguistique n'a qu'à être appliqué par l'hémisphère gauche, et ce d'autant plus que ce système est utilisé ou routinisé. Par contre, il est possible que toutes les langues ne présentent pas le même degré de systématibilité. Par exemple, dans les langues, tel le chinois, dont le versant écrit repose sur des idéogrammes plutôt que sur un code littéral-graphémique, le système descriptif requis pour la réalisation d'une tâche de lecture (décodage) diffère probablement. En effet, le recours à un code littéralgraphémique repose probablement sur un système descriptif très "systématisable " car le décodage du langage écrit peut représenter l'application d'un code de correspondance grapho-phonémique. Par contre, le recours à des idéogrammes représente un système descriptif en soi, qui ne semble pas correspondre à l'application d'un code de correspondance avec le versant oral (Paradis 1983). Une telle hypothèse trouve peut-être certains appuis dans le fait que la survenue d'une lésion hémisphérique gauche semble moins systématiquement perturber le fonctionnement linguistique chez ceux dont le versant écrit passe par une représentation idéographique (Bradshaw et Nettleton 1983 ; Hagiwara 1983). Il est donc permis de penser que toutes les langues ne présentent pas nécessairement le même degré de systémabilité et que, conséquemment, les réseaux neuroniques sous-tendant chaque langue puissent être plus ou moins latéralisés selon la systématibilité d'une langue donnée.

Si on ajoute à un tel concept la notion de routinisation, le modèle de Goldberg et Costa peut nous amener à faire quelques prédictions quant à l'organisation cérébrale de ceux qui maîtrisent plus d'une langue. Chez les bilingues ou polyglottes, la latéralisation et l'organisation des réseaux neuroniques susceptibles de sous-tendre chacune des langues pourraient varier, entre autres, selon la systématibilité de la langue et son degré d'utilisation. Le réseau neuronique responsable sera d'autant plus latéralisé et concentré au sein de l'hémisphère gauche que la langue peut faire l'objet d'un système descriptif systématique et univoque ${ }^{2}$ et qu'elle a fait l'objet d'une routinisation, c'est-à-dire d'une utilisation répétée. Une telle prédiction trouve certains appuis dans l'étude des aphasies chez les bilingues et/ou polyglottes. Suite à une revue exhaustive de la question, Vaid et Genesee (1980) concluent que l'organisation fonctionnelle pour la, ou les, langue(s) seconde(s) est d'autant moins latéralisée à gauche que (a) la langue seconde est apprise

2. Le cas du japonais, discuté dans l'article précédent, peut servir d'exemple. 
tardivement, et que (b) l'exposition à cette langue seconde n'est pas systématique. Dans le contexte du modèle de Goldberg et Costa, le premier point signifie une faible routinisation et le second, un faible degré de systématisation de la langue seconde.

Un raisonnement similaire pourrait être fait à propos de l'acte de traduction. En effet, traduire un segment de langage d'une langue 1 à une langue 2 est une tâche qui nécessite l'application d'un système descriptif, d'un code, qui permet la transposition de 1 en 2 d'un contenu donné. Il est possible que le système qui permet le passage d'une langue à une autre soit plus ou moins systématisable. Par exemple, si les deux langues ont une structure comparable, tels l'espagnol et l'italien, il est possible que l'acte de traduction puisse être fortement systématisable et reposer essentiellement sur l'application d'un système de conversion d'une langue à l'autre. Par contre, si les deux langues ont des structures très dissemblables, tels le français et le suédois ou encore le français et le vietnamien, il est probable que l'acte de traduction soit moins systématisable, c'est-àdire qu'il repose moins sur l'application d'un système de conversion d'une langue à l'autre mais plutôt sur l'extraction du contenu de la première langue et sa remise en forme dans la seconde langue. Bien sûr, ce ne sont là que des spéculations mais si tel était le cas, l'organisation fonctionnelle du cerveau des traducteurs pourrait bien dépendre, outre de facteurs discutés dans le cadre du chapitre précédent, du degré de systématibilité de l'acte, ou des actes de traduction qu'ils sont amenés à faire, ainsi que de leur degré de routinisation (voir Nespoulous de même que Le Dorze et Nespoulous, dans ce même numéro).

Comme nous l'avons évoqué précédemment, un tel modèle ne concerne pas que la fonction linguistique. Il concerne l'ensemble des fonctions cognitives. L'étude de la fonction musicale, par exemple, offre des données qui sont loin d'être incompatibles avec le modèle présenté. L'un des paramètres particulièrement intéressant à ce sujet est la comparaison entre " novices" et " experts", autrement dit, entre ceux qui abordent la musique sans système descriptif particulier (" novices ") et ceux qui, au contraire, possèdent l'ensemble des règles du langage musical et, qui plus est, ont eu l'occasion de les routiniser (" experts"). Malgré quelques contradictions, les résultats des études utilisant la technique de présentation de stimuli musicaux en écoute dichotique chez des sujets normaux sont, à cet égard, intéressants. Dans ces études, la procédure expérimentale consiste à présenter simultanément deux stimuli auditifs, l'un à chacune des oreilles ; bien que l'organisation centrale du système auditif soit bilatérale, il existe dans cette condition une certaine prévalence fonctionnelle des voies controlatérales sur les voies ipsilatérales, prévalence qui ne se mesure qu'auprès de groupes de sujets. L'écoute dichotique de stimuli verbaux fait ressortir, au niveau d'un groupe, une relative prévalence des stimuli présentés à l'oreille droite (hémisphère gauche). Utilisant des stimuli musicaux, Bever et Chiarello (1974) et Johnson (1977) ont rapporté une prévalence de l'oreillle gauche (hémisphère droit) chez un groupe de sujets " novices " mais une prévalence inverse - c'est-à-dire de la gauche sur la droite - chez un groupe de musiciens "experts". Autrement dit, l'organisation pour la fonction musicale serait sous la dépendance de réseaux neuroniques impliquant plus l'hémisphère droit que le gauche chez des "novices" de la musique ; à l'inverse, l'accès à un système descriptif des règles du langage musical combiné à sa routinisation chez les musiciens "experts " ferait en sorte que cette organisation fonctionnelle serait plutôt sous la dépendance de réseaux neuroniques impliquant plus l'hémisphère gauche que le droit. L'étude des effets de lésions cérébrales acquises sur la fonction musicale de musiciens experts est une autre source intéressante de données. Par exemple, Théophile Alajouanine (1948) rapporte l'observation de ce grand compositeur français - Maurice Ravel - devenu aphasique par lésion gauche qui pouvait toujours reconnaître et même apprécier la qualité artistique d'une oeu- 
vre mais qui était incapable de composer. Une telle observation s'intègre bien dans le cadre du modèle de Goldberg et Costa puisque l'impossibilité de composer pourrait représenter l'atteinte des réseaux neuroniques de l'hémisphère gauche susceptibles de sous-tendre le système descriptif nécessaire à la mise en forme d'impressions musicales.

Un dernier exemple d'application du modèle de Goldberg et Costa aura trait à la reconnaissance des visages. La perte de cette fonction liée à la survenue d'une lésion cérébrale - la prosopagnosie - ne s'observe que dans les cas où la lésion implique les deux hémisphères (Sergent, 1982), ou tout au moins l'hémisphère droit (Poncet 1983). Il n’a jamais été rapporté de prosopagnosie faisant suite à une lésion limitée à l'hémisphère gauche. Une telle situation tient peut-être au fait que la reconnaissance des visages n'est pas une activité qui s'effectue normalement par le biais de l'application d'un système descriptif. Une telle hypothèse est d'autant plus vraisemblable que l'une des façons de pallier une prosopagnosie, pour ceux qui en sont atteints, est justement d'avoir recours à un système descriptif telle la recherche de signes particuliers (e.g. cicatrices, couleur des yeux). Par contre, compte tenu de ce qui a été exposé précédemment à propos de la différence entre novices et experts, il est permis d'émettre l'hypothèse selon laquelle l'organisation fonctionnelle du cerveau pour la reconnaissance des visages serait différente chez les portraitistes. En effet, ces derniers doivent probablement avoir recours à un système descriptif car, dans leur cas, il s'agit de reproduire un visage. Ainsi, selon le modèle présentement discuté, l'hémisphère gauche pourrait bien sous-tendre les processus nécessaires au système descriptif de reproduction des visages. Le facteur " reproduction "pourrait ainsi être susceptible de favoriser le recours à un système descriptif dans la réalisation d'une tâche donnée. Si tel était le cas, un portraitiste pourrait bien devenir prosopagnosique, ou tout au moins incapable de faire des portraits à la suite d'une lésion cérébrale gauche.

Si des modèles de fonctionnement cérébral tel celui de Goldberg et Costa se vérifiaient, toute recherche des relations mutuelles entre cerveau et comportement - par conséquent, entre cerveau et langage - devrait à l'avenir prendre en compte la nature exacte d'une tâche donnée, la stratégie utilisée par le sujet de même que le degré de routinisation de cette tâche pour ce sujet. Comme nous l'avons vu, il existe habituellement un lien entre les degrés de routinisation et de systématibilité d'une tâche : plus une tâche est routinisée, plus les chances sont grandes qu'elle ait préalablement fait l'objet de l'application d'un système descriptif donné (e.g., la fonction linguistique, la fonction musicale pour les experts). Finalement, il a été brièvement évoqué qu'une tâche aura plus de chances de faire l'objet d'un système descriptif si elle implique la reproduction d'une activité donnée (e.g., le portraitiste).

\section{LES DIFFÉRENCES INTERINDIVIDUELLES}

Nous avons vu, jusqu'à présent, que l'étude des relations mutuelles entre cerveau et langage ne peut désormais que difficilement faire fi de certaines caractéristiques spécifiques tant à une tâche donnée qu'aux relations mutuelles existant entre un individu donné et cette tâche, le tout placé dans sa dimension chronobiologique. Voilà un ensemble de connaissances qui, si elles devaient toutes être prises en considération, pourraient signifier que chaque individu présente une organisation fonctionnelle du cerveau qui lui est pratiquement propre, organisation procédant de l'ensemble des faits susmentionnés.

L'une des façons d'aborder la question des différences interindividuelles est celle retenue par un sous-ensemble de la psychologie cognitive. Bien que la prise en compte des différences interindividuelles en psychologie remonte à ses tous débuts, l'importance des connaissances accumulées ces dernières années en psychologie cognitive a fait se dé- 
tacher ce sous-ensemble qui focalise son attention sur le problème des différences interindividuelles dans le fonctionnement cognitif (e.g., Dillon et Schmeck 1983). Comme le mentionnent Lohman et Kyllonen (1983), la plupart des études en psychologie cognitive présumaient - et présument encore pour la plupart - que tout sujet mis devant une tâche à effectuer l'accomplira de la même façon. Or, ce n'est pas parce que deux sujets obtiennent les mêmes performances à une tâche qu'ils l'ont nécessairement réalisée de la même façon, en utilisant la, ou les, même(s) stratégie(s) cognitive(s). Déjà en 1938, Thurstone avait émis l'opinion que la seule analyse des résultats, ou des corrélations entre différents résultats, pouvait obscurcir l'éventuelle présence de différences interindividuelles dans le choix de la stratégie de solution d'une tâche donnée.

On dispose aujourd'hui d'un ensemble d'informations qui montrent bien que la réalisation d'une tâche donnée ne passe pas nécessairement par la mise en oeuvre d'une seule et même stratégie de solution (Dilon et Schmeck 1983). Les premières de ces informations nous proviennent des sujets eux-mêmes ; en effet, la seule introspection - méthodologie limitée mais utile - a permis de soupçonner fortement la présence de telles différences interindividuelles (Lohman et Kyllonen 1983). Les résultats d'approches plus expérimentales nous permettent aujourd'hui d'affirmer l'existence de telles différences, tout au moins dans les cas des fonctions spatiales et verbales. Par exemple, Pellegrino et Goldman (1983) ont démontré l'existence de stratégies différentes pour la réalisation d'une tâche de rotation mentale d'un objet, les unes probablement purement spatiales, les autres faisant probablement intervenir une médiation verbale. Il appert de plus que le recours d'un individu donné à une stratégie donnée n'implique pas nécessairement que cet individu se caractérise par le recours à une et une seule stratégie. Les stratégies utilisées semblent varier avec l'âge, chez un même individu, et dépendre fortement de la nature de la tâche et de son degré de complexité : plus une tâche est difficile, plus la variété de stratégies possibles pour résoudre cette tâche est grande et imprévisible (Lochman et Kyllonen 1983). Ces derniers faits tendent à minimiser un certain courant de pensée qui espérait caractériser chaque individu par le recours à une et une seule stratégie de solution, autrement dit par un "style cognitif " (Witkin et Goodenough 1980). Au contraire, selon Lohman et Kyllonen (1983), les stratégies de solution d'une tâche donnée sont tributaires à la fois de l'individu et de la tâche. D'une part, tenter de caractériser les individus par un style cognitif présuppose que le style cognitif soit une caractéristique immuable de la personne. D’autre part, classifier les tâches selon le type de stratégie nécessaire à sa solution présuppose que tous les individus solutionnent cette tâche de la même manière. Il est plutôt nécessaire de faire ressortir les interactions entre les individus et les tâches devant lesquelles ils sont placés afin de pouvoir éventuellement rendre compte de différences interindividuelles qui sont à la fois tributaires de l'individu et de la tâche. Voilà une autre dimension que l'étude des relations mutuelles entre cerveau et langage devrait dorénavant prendre en considération.

Nous avons ici tenté d'entrevoir quelques-unes des nouvelles perspectives à considérer dans l'étude des relations - de plus en plus complexes - entre le cerveau et l'une de ses fonctions cognitives, le langage. Les quelques nouvelles dimensions que nous avons brièvement évoquées nous semblent susceptibles de contribuer à une meilleure et plus juste conception de l'organisation fonctionnelle du cerveau pour le langage. Bien sûr, l'avenir nous réserve probablement l'identification d'autres dimensions encore que nous ne soupçonnons même pas aujourd'hui, tant au niveau fonctionnel qu'au niveau neurobiologique. En même temps, certaines des nouvelles dimensions que nous entrevoyons actuellement s'avéreront peut-être dans l'avenir comme non pertinentes. Dans tous les cas, il faut agir avec prudence et patience. 
BIBLIOGRAPHIE

ALAJOUANINE, Th. (1948): "Aphasia and Artistic Realization", Brain, 71, pp. 229-241.

ALAJOUANINE, Th. (1968) : l'Aphasie et le langage pathologique, Paris, Baillière et Fils.

ALAJOUANINE, Th. \& F. LHERMITTE (1965): "Acquired Aphasia in Children", Brain, 88, pp. 653-662.

BASSER, L.S. (1962) : "Hemiplegia of Early Onset and the Faculty of Speech with Special Reference to the Effects of Hemispherectomy", Brain 85, pp. 428-460.

BEVER, T.G. \& K. CHIARELLO (1974) : "Cerebral Dominance in Musicians and Non-Musicians", Science, 185 , pp. 537-539.

BRADSHAW, J.L. \& N.C. NETTLETON (1983) : Human Cerebral Asymmetry, Englewood Cliffs, New Jersey, Prentice-Hall Inc.

BROWN, J.W. (1976) : "The Neural Organization of Language : Aphasia and Lateralization", Brain and Language, 3 , pp. $482-494$

BROWN, J.W. (1982) : "Hierarchy and Evolution in Neurolinguistics", in Neural Models of Language Processes, New York, Academic Press.

BROWN, J.W. \& H. HÉCAEN (1976) : "Lateralization and Language Representation", Neurology, 26, pp. 183-189.

BRYDEN, M.P. (1979) : "Evidence for Sex-Related Differences in Cerebral Organization", in M. WITTIG \& A.C. PETERSEN (Edits), Sex-Related Differences in Cognitive Functioning : Developmental Issues, New York, Academic Press.

DILLON, R.F. \& R.R. SCHMECK (Edits) (1983) : Individual Differences in Cognition, Vol. 1, New York, Academic Press.

GOLDBERG, E. \& L.D. COSTA (1981) : "Hemisphere Differences in the Acquisition and Use of Descriptive Systems", Brain and Language, 14, pp. 144-173.

HAGIWARA, H. (1983) : "Dissociations between Reading Syllabic and Ideographic Script in Japanese Aphasic Patients", paper presented at BABBLE, an annual conference reporting research in the neuropsychology of language, Niagara Falls.

HÉCAEN, H. (1976) : "Acquired Aphasia in Children and the Ontogenesis of Hemispheric Functional Specialization", Brain and Language, 3, pp. 114-134.

JOANETTE, Y., A. ALI-CHÉRIF, F. DELPUECH, M. HABIB, J.-F. PÉLISSIER \& M. PONCET (1983) : "Évolution de la sémiologie aphasique avec l'âge. Discussion à propos d'une observation anatomoclinique ", Revue neurologique (Paris), 139, 11, pp. 657-664.

JOHNSON, P.R. (1977) : "Dichotically-Stimulated Ear Differences in Musicians and Nonmusicians", Cortex, 13, pp. 385-389.

KIMURA, D. (1980) : "Sex differences in Intrahemispheric Organization of Speech", The Behavioral and Brain Sciences, 3, pp. 240-241.

LECOURS, A.R., E. OSBORN, L. TRAVIS, F. ROUILLON \& G. LAVALLÉE-HUYNH (1981) "Jargons", in J.W. BROWN (Edit.), Jargonaphasia, Chapter 2, pp. 9-38, New York, Academic Press.

LEVY, J. (1972) : "Lateral Specialization of the Human Brain : Behavioral Manifestations and Possible Evolutionary Basis", In J.A. KIGER (Edit.), The Biology of Behavior, Corvallis, Ore., Oregon State University Press.

LOHMAN, D.F. \& P.C. KYLLONEN (1983) : "Individual Differences in Solution Strategy on Spatial Tasks", in R.F. DILLON \& R.R. SCHMECK (Edits), Individual Differences in Cognition, Vol. 1, Chapter 4, pp. 105-135, New York, Academic Press.

McGlonE, J. (1980) : "Sex Differences in Human Brain Organization : A critical Survey", The Behavioral and Brain Sciences, 3, pp. 215-227.

OBLER, L.K., M.L. ALBERT, H. GOODGLASS \& D.F. BENSON (1978) : "Aphasia Type and Aging", Brain and Language, 6, pp. 318-322.

PARADIS, M. (1983) "Aspectcs of the Japanese Writing System Relevant to Psycho- and Neurolinguistic Studies", paper presented at BABBLE, an annual conference reporting research in the neuropsychology of language, Niagara Falls.

PELLEGRINO, J.W. \& S.R. GOLDMAN (1983) : "Developmental and Individual Differences in Verbal and Spatial Reasoning", in R.F. DILLON \& R.R. SCHMECK (Edits), Individual Differences in Cognition, Vol. 1, Chapter 5, pp. 137-180, New York Academic Press.

PERECMAN, E. \& J.W. BROWN (1981): "Phonemic Jargon : A Case Report", in J.W. BROWN (Edit.), Jargonaphasia, Chapter 10, pp. 177-257, New York, Academic Press.

PONCET, M. (1983) : Communication personnelle.

PONCET, M. \& J. PONZIO (1975) : "Les troubles de la communication du langage chez les sujets du troisième âge ", communication présentée aux Journées d'étude sur la pathologie du troisième âge, Marseille.

SEGALOWITZ, S.J. \& M.P. BRYDEN (1983) : "Individual Differences in Hemispheric Representation of 
Language", in S.J. SEGALOWITZ (Edit.), Language Functions and Brain Organization, Chapter 15, pp. 341-372, New York, Adademic Press.

SERGENT, J. (1982) : "About Face : Left-Hemisphere Involvement in Processing Physiognomies", Journal of Experimental Psychology : Human Perception and Performance, 8, 1, pp. 1-14.

THURSTONE, L.L. (1983) : "The Perceptual Factor", Psychometrica, 3, pp. 1-12.

VAID, J. \& F. GENESEE (1980) : "Neuropsychological Approaches to Bilingualism", Canadian Journal of Psychology, 34, pp. 417-445.

VARGHA-KHADEM, F., A. O'GORMAN \& G.V. WATTERS (1983) : "Aphasia in Children with 'Prenalal' vs Postnatal Left Hemisphere Lesions : A Clinical and CT Scan Study", paper presented at the 11 th meeting of the International Neuropsychological Society, Mexico City.

WITKIN, H.A. \& D.R. GOODENOUGH (1980) : "Cognitive Styles : Essence and Origins - FieldDependence and Field-Independence". Psychological Issues Monograph, 51, p. 130. 